\title{
Model Predictive Controller for a Planar Tensegrity Mechanism with decoupled position and stiffness control.
}

JR Jurado Realpe, Salih Abdelaziz, and Philippe Poignet.

\begin{abstract}
Precise trajectory tracking and stiffness modulation for tensegrity mechanisms are a challenging topic that can open new horizon of applications for this type of systems. This paper presents a new control strategy of tensegrity mechanisms using a model predictive controller (MPC). Based on a dynamic model, the proposed approach allows to track trajectories with low and relatively high dynamics as well as to modulate the mechanism stiffness by changing only the controller's parameters. Trajectories of $30 s, 5 s$ and $1 s$ are performed showing a trajectory tracking improvement of up to $64 \%$ in the root mean square error when compared to literature results.
\end{abstract}

Key words: Dynamic modeling, MPC controller, Stiffness modulation,Tensegrity mechanism, Trajectory tracking.

\section{Introduction}

The word tensegrity comes from the contraction of tensional and integrity [1]. A tensegrity system is composed by compressive and tensile elements that interacts to define a stable volume [2]. The integrity of the structure is then ensured by only the internal forces produced by the tensile elements (cables, springs). Their large workspace to size ratio, their light weight and their ability to modulate stiffness, make tensegrity systems an interesting solution for structural applications [3] [4], deployable systems [5] [6], mobile robots [7] [8] or manipulators [9] [10].

Different control approaches have been proposed to control the motion of tensegrity mechanisms. The authors are commonly focused in the generation of gait for locomotion using open loop controllers [11] [12], neuronal networks [13] [14], kinematic models [10] or Lyapunov function [15]. Nevertheless, the trajectory tracking

The Autors are with the LIRMM, Université de Montpellier, CNRS, France.

e-mail: [ juradoreal, abdelaziz, poignet ]@lirmm.fr 
has not been a priority. To our knowledge, only two works [10] [16] propose strategies to control finely trajectory tracking. On one hand, a PID controller and a tension distribution algorithm [10], inspired from cable-driven robots controllers, has been proposed to control the position and the stiffness of a 1 DOF planar tensegrity mechanism. This controller presents good position tracking for trajectories with low dynamic but loose performance when tracking relatively high dynamics trajectories. On the other hand, a Model Predictive Controller (MPC) with inverse static optimization has been introduced to realize the trajectory tracking of a tensegrity spine robot. Nevertheless, stiffness modulation is not addressed.

Our interest here is to fully exploit a tensegrity system by controlling its position and stiffness. A dynamic-based control strategy would be pertinent to deliver good trajectory tracking performance. A MPC using a dynamic model is here proposed to modulate the stiffness and trajectory tracking of the mechanism in an asynchronous way by only modifying the control parameters of the controller. The proposed strategy is experimentally validated on a 1 DOF planar cable-driven tensegrity mechanism.

This paper is organized as follow. The static, stiffness and dynamic models of the planar tensegrity mechanism is developed in section 2 . Then a MPC strategy is proposed in section 3. Position tracking and stiffness modulation results are discussed in section 4. Finally conclusions and future work are presented in section 5.

\section{System modelling}

The system studied here is a 1 DOF planar tensegrity mechanism, whose end effector is considered as the bar bc (Fig 1). The end effector orientation is denoted $\theta$. The structure is composed by an articulated equilateral parallelogram, of length $L$, and is driven by two cables. Each cable is attached to one corner of the parallelogram at one end and to a linear spring, of stiffness $k$, on the other end. The mechanism is actuated by two actuators. The angular position for each motor $i$ is denoted $\alpha_{i}$. Pulleys, of radius $R$, are mounted on the actuators so that $\rho_{i}=R \alpha_{i}$ describes the displacement of the cable $i$. This redundancy of actuation is performed so as to allow stiffness modulation of the system.

\subsection{Static and stiffness models}

The static model of the system is computed using the virtual work approach. It is described as:

$$
\mathbf{W} \tau=\Gamma
$$

where $\mathbf{W}=[L \sin (\theta / 2),-L \cos (\theta / 2)] . \Gamma$ denotes the torque generated by the two cables on the end effector. The components of the vector $\tau=\left[\tau_{1}, \tau_{2}\right]^{T}$ describe the 

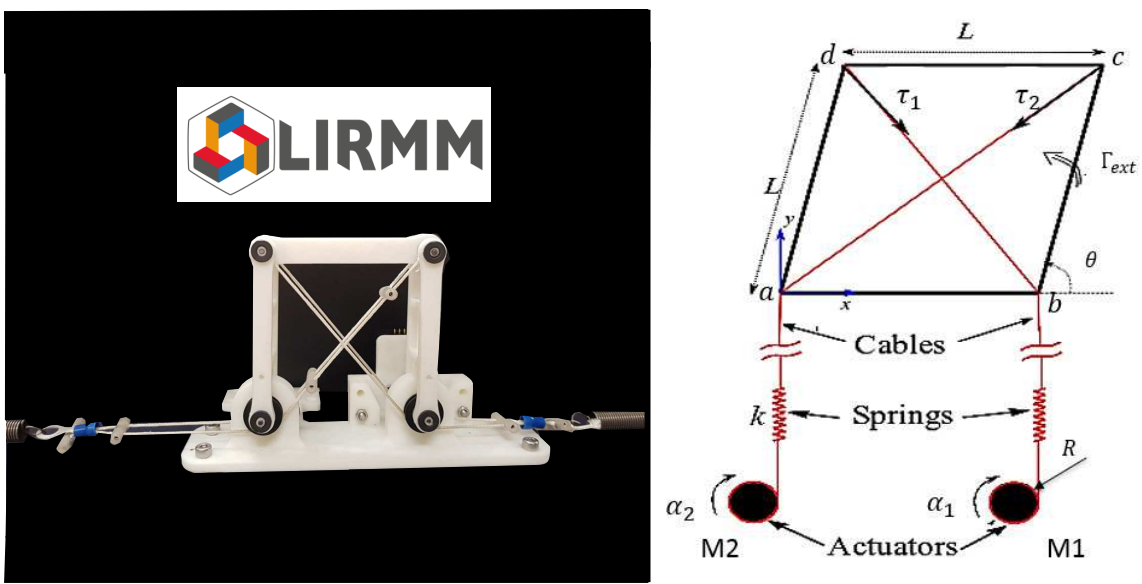

Fig. 1 planar mechanimt.

cables tensions, computed as:

$$
\left\{\begin{array}{l}
\tau_{1}=k \Delta l_{1}=k\left(\rho_{1}+2 L \cos (\theta / 2)-L \sqrt{2}\right) \\
\tau_{2}=k \Delta l_{2}=k\left(\rho_{2}+2 L \sin (\theta / 2)-L \sqrt{2}\right)
\end{array}\right.
$$

where $\Delta l_{i}$ denotes the elongation of the spring $i$. The potential energy $U$ of the system is assumed to be only the elastic energy stored in the springs:

$$
U=\frac{1}{2} k\left(\Delta l_{1}^{2}+\Delta l_{2}^{2}\right)
$$

The static equilibrium of the platform is obtained by solving $\frac{\partial U}{\partial \theta}=0$ :

$$
\theta=2 \arctan \left(\frac{L \sqrt{2}-\rho_{2}}{L \sqrt{2}-\rho_{1}}\right)
$$

The angular stiffness $K_{\theta}$ at the equilibrium configuration is obtained by:

$$
K_{\theta}=\frac{\partial^{2} U}{\partial \theta^{2}}=-\frac{\partial \Gamma}{\partial \theta}=-\frac{L}{2}\left(\cos (\theta / 2) \tau_{1}+\sin (\theta / 2) \tau_{2}\right)+k L^{2}
$$

\subsection{Dynamic model}

The dynamic model of the structure is calculated using Euler-Lagrange formulation. The total kinetic energy $E_{k}$ of the system is defined by: 


$$
E_{k}=\frac{5}{6} m L^{2} \dot{\theta}^{2}
$$

where $m$ denotes the mass of one bar of the parallelogram. The equation of motion is therefore given by:

$$
\mathrm{f}_{\mathrm{d}}+\mathrm{f}_{\mathrm{q}}+\Gamma_{\text {ext }}=\frac{\mathrm{d}}{\mathrm{dt}} \frac{\partial \mathscr{L}}{\partial \dot{\theta}}-\frac{\partial \mathscr{L}}{\partial \theta}
$$

where $\Gamma_{\text {ext }}$ denotes the external torque applied on the end-effector and $\mathscr{L}=E_{T}-U$. In this study, the friction and the gravity forces, respectively, $\mathrm{f}_{\mathrm{d}}$ and $\mathrm{f}_{\mathrm{q}}$, are neglected. The structure dynamics is therefore described as:

$$
\Gamma_{\text {ext }}=\frac{5}{3} m L^{2} \ddot{\theta}+\frac{\partial U}{\partial \theta}
$$

The dynamic of the actuator $i$ is defined using Newton's law:

$$
\mathrm{C}_{\mathrm{i}}-\mathrm{C}_{\mathrm{v}} \dot{\alpha}_{i}-R \tau_{i}=\mathrm{I}_{\mathrm{m}} \frac{\mathrm{d} \dot{\alpha}_{i}}{\mathrm{dt}}
$$

with $\mathrm{C}_{\mathrm{i}}$ and $\mathrm{C}_{\mathrm{v}}$ being respectively the torque and viscous coefficient of the actuator. Finally, the complete dynamic model of the tensegrity mechanism is expressed in a matrix formulation as:

$$
\left(\begin{array}{l}
R \mathrm{C}_{1} \\
R \mathrm{C}_{2} \\
\Gamma_{\text {ext }}
\end{array}\right)=\left(\begin{array}{ccc}
\mathrm{I}_{\mathrm{m}} & 0 & 0 \\
0 & \mathrm{I}_{\mathrm{m}} & 0 \\
0 & 0 & \frac{5 m L^{2}}{3}
\end{array}\right)\left(\begin{array}{c}
\ddot{\rho}_{1} \\
\ddot{\rho_{2}} \\
\ddot{\theta}
\end{array}\right)+\left(\begin{array}{c}
\mathrm{C}_{\mathrm{v}} \dot{\rho}_{1} \\
\mathrm{C}_{\mathrm{v}} \dot{\rho}_{2} \\
0
\end{array}\right)+\left(\begin{array}{c}
R^{2} \tau_{1} \\
R^{2} \tau_{2} \\
\frac{\partial U}{\partial \theta}
\end{array}\right)
$$

\section{Proposed control strategy}

A control approach that allows to asynchronously track low/high dynamic trajectories and modulate the stiffness of the tensegrity mechanism is hereafter presented. It consists on a linear model predictive controller based on a discrete-time state-space model.

\subsection{Dynamic model linearization}

The linearization of (10) is performed around an equilibrium point $\left(\rho_{10}, \rho_{20}, \theta_{0}\right)$. Only $\partial U / \partial \theta, \tau_{1}$ and $\tau_{2}$ present nonlinear terms. The linearized terms are computed using the first-order Taylor expansion as:

$$
\left(\begin{array}{l}
\tau_{1} \\
\tau_{2}
\end{array}\right)=\left(\begin{array}{l}
k \rho_{1}-f_{1} \theta+f_{3} \\
k \rho_{2}+f_{2} \theta+f_{4}
\end{array}\right)
$$


Title Suppressed Due to Excessive Length

$$
\frac{\partial U}{\partial \theta}=-f_{1} \rho_{1}+f_{2} \rho_{2}-f_{5} \theta-f_{6}
$$

such that:

$f_{1}=k L \sin \left(\theta_{0} / 2\right)$

$f_{2}=k L \cos \left(\theta_{0} / 2\right)$

$f_{3}=2 f_{2}+f_{1} \theta_{0}-k L \sqrt{2}$

$f_{4}=2 f_{1}-f_{2} \theta_{0}-k L \sqrt{2}$

$f_{5}=-L \sqrt{2} / 2\left(f_{1}+f_{2}\right)+f_{1} \rho_{20} / 2+f_{2} \rho_{10} / 2$

$f_{6}=L \sqrt{2}\left(f_{2}-f_{1}\right)+\left\{L \sqrt{2} / 2\left(f_{1}+f 2\right)-\left(f_{1} \rho_{20} / 2+f_{2} \rho_{10} / 2\right)\right\} \theta_{0}$

By defining the state vector $\mathbf{x}=\left[\rho_{1}, \rho_{2}, \theta, \dot{\rho}_{1}, \dot{\rho}_{2}, \dot{\theta}\right]^{T}$ and the control vector as the torque of the motors $\mathbf{u}=\left[\mathrm{C}_{1}, \mathrm{C}_{2}\right]^{T}$ and by using the linearized expressions, the continuous-time state-space is expressed as:

$$
\dot{\mathbf{x}}=\mathbf{A}_{c} \mathbf{x}+\mathbf{B}_{c} \mathbf{u}+\mathbf{d}_{c}
$$

with:

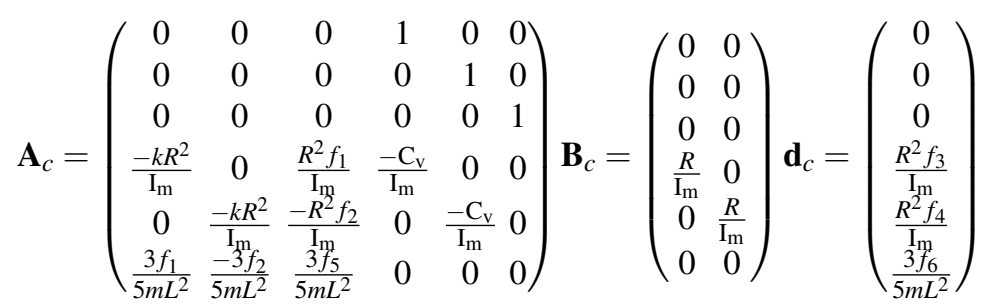

The linear discrete-time model is defined as:

$$
\mathbf{x}_{k+1}=\mathbf{A}_{k} \mathbf{x}_{k}+\mathbf{B}_{k} \mathbf{u}_{k}+\mathbf{d}_{k}
$$

\subsection{MPC formulation}

The objective of the MPC controller is to minimize the error $\mathbf{E}_{k}$ over an horizon $\mathrm{H}_{\mathrm{p}}$ between the desired trajectory $\mathbf{T}_{k}=\left[\mathbf{x}_{k+1}^{d}, \mathbf{x}_{k+2}^{d}, \ldots, \mathbf{x}_{k+\mathrm{H}_{\mathrm{p}}}^{d}\right]^{T}$ and the predicted output $\mathbf{Y}_{k}=\left[\mathbf{x}_{k+1}, \mathbf{x}_{k+2}, \ldots, \mathbf{x}_{k+\mathrm{H}_{\mathrm{p}}}\right]^{T}$. The cost function is defined as:

$$
\mathbf{V}_{k}=\mathbf{E}_{k}^{T} \mathbf{Q} \mathbf{E}_{k}+\Delta \mathbf{U}_{k}^{T} \mathbf{R} \Delta \mathbf{U}_{k}
$$

with $\mathbf{E}_{k}=\mathbf{T}_{k}-\mathbf{Y}_{k}$. $\mathbf{Q}$ and $\mathbf{R}$ are diagonal weight matrices.

In order to solve the predictive control problem, it is necessary to predict the future states $\mathbf{x}_{k+i}, i=1, \ldots, \mathrm{H}_{\mathrm{p}}$. To do so, we consider that over a horizon $\mathrm{H}_{\mathrm{p}}$ the linear time varying system (15) can be approximated by a linear invariant system. This yields to consider the matrices $\mathbf{A}_{k}, \mathbf{B}_{k}$ and $\mathbf{d}_{k}$ as constants, which mean that $\mathbf{A}_{k+i}=\mathbf{A}_{k}=\mathbf{A}, \mathbf{B}_{k+i}=\mathbf{B}_{k}=\mathbf{B}$ and $\mathbf{d}_{k+i}=\mathbf{d}_{k}=\mathbf{d}$ for $i=1, \ldots, \mathrm{H}_{\mathrm{p}}$. With this as- 
sumption the future states can be computed by applying $\mathrm{H}_{\mathrm{p}}$ times the equation (15):

$$
\begin{aligned}
& \mathbf{Y}_{k}=\left(\begin{array}{c}
\mathbf{x}_{k+1} \\
\mathbf{x}_{k+2} \\
\vdots \\
\mathbf{x}_{k+\mathrm{H}_{\mathrm{p}}}
\end{array}\right)=\underbrace{\left[\begin{array}{c}
\mathbf{A} \\
\mathbf{A}^{2} \\
\vdots \\
\mathbf{A}^{\mathrm{H}_{\mathrm{p}}}
\end{array}\right]}_{\Psi} \mathbf{x}_{k}+\underbrace{\left[\begin{array}{c}
\mathbf{B} \\
\mathbf{A B}+\mathbf{B} \\
\vdots \\
\sum_{i=0}^{\mathrm{H}_{\mathrm{p}}-1} \mathbf{A}^{i} \mathbf{B}
\end{array}\right]}_{\gamma} \mathbf{u}_{k-1}+\underbrace{\left[\begin{array}{c}
\mathbf{d} \\
\mathbf{A d}+\mathbf{d} \\
\vdots \\
\sum_{i=0}^{\mathrm{H}_{\mathrm{p}}-1} \mathbf{A}^{i} \mathbf{d}
\end{array}\right]}_{\mathbf{D}}+\ldots
\end{aligned}
$$

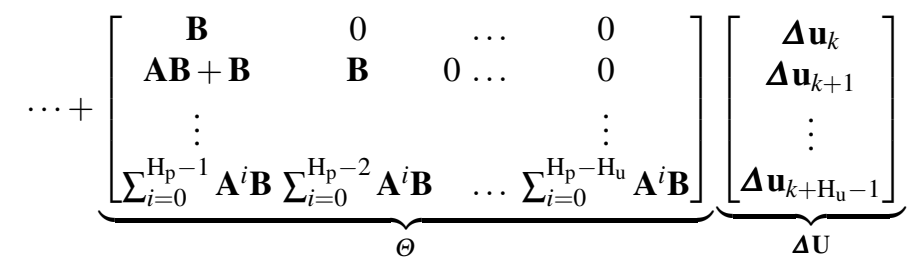

To take into account the unilateral tensions in the cables, a constrained optimization problem has to be considered. As the control variables of the MPC controller are the torques of the actuators, the tension limits, $\tau_{\min }=\left[\tau_{\min }, \tau_{\min }\right]^{T}$ and $\tau_{\max }=\left[\tau_{\max }, \tau_{\max }\right]^{T}$, have to be converted into torques limits $\mathbf{u}_{\min }=\left[\mathrm{C}_{\min }, \mathrm{C}_{\min }\right]^{T}$ and $\mathbf{u}_{\max }=\left[\mathrm{C}_{\max }, \mathrm{C}_{\max }\right]^{T}$. The solution of (16) can be obtained by solving the constrained quadratic programming $(\mathrm{QP})$ problem:

$$
\begin{array}{r}
\min _{\boldsymbol{\Delta} \mathbf{U}} \boldsymbol{\Delta} \mathbf{U}^{T} \mathbf{H} \boldsymbol{\Delta} \mathbf{U}+\mathbf{G}^{T} \boldsymbol{\Delta} \mathbf{U} \\
\text { subject to : } \mathbf{u}_{\min } \leq \mathbf{u} \leq \mathbf{u}_{\max }
\end{array}
$$

with $\mathbf{H}=\Theta^{T} \mathbf{Q} \Theta+\mathbf{R}$ and $\mathbf{G}=2 \Theta^{T} \mathbf{Q}\left(\mathbf{T}_{k}-\Psi \mathbf{x}_{k}-\gamma \mathbf{u}_{k-1}-\mathbf{D}\right)$. This problem has to be solved at each step $k$. Once the optimal solution $\boldsymbol{\Delta} \mathbf{U}$ is found, the first control output is applied and kept until the next time step.

\subsubsection{Trajectory tracking}

The overall control approach is composed of an external MPC controller and an internal tension control loop (cf. Fig2). The control output $\mathbf{u}_{k}$ of the MPC is converted into a desired tension $\tau^{d}$. This vector of tension is then compared to $\tau^{m}$ that contains the real tensions in the cables. These tensions are measured using force sensors. A tension controller $\mathbf{C}_{\tau}$ is considered to control these tensions in the inner loop. The output of this tension controller is sent to the actuators as desired velocities $\dot{\boldsymbol{\alpha}}^{d}=\left[\dot{\alpha}_{1}^{d}, \dot{\alpha}_{2}^{d}\right]^{T}$.

During the trajectory tracking, the variables $\theta$ and $\dot{\theta}$ in the state vector $\mathbf{x}$ are to be controlled. To do so, the weight matrix $\mathbf{Q}$ has to be defined with high values for the components $\mathrm{Q}_{\theta}$ and $\mathrm{Q}_{\dot{\theta}}$ and values near to zero for the other components $\mathrm{Q}_{\rho_{1}}, \mathrm{Q}_{\rho_{2}}, \mathrm{Q}_{\dot{\rho}_{1}}$. and $\mathrm{Q}_{\dot{\rho}_{2}}$ : 
Title Suppressed Due to Excessive Length

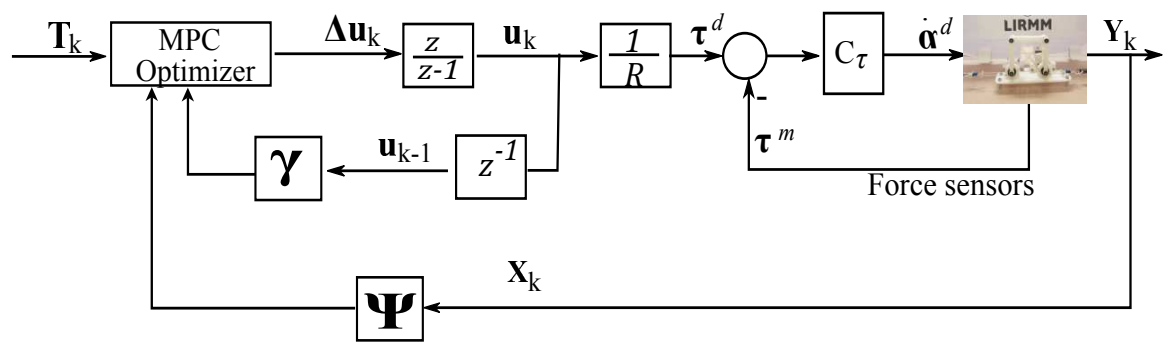

Fig. 2 Strategy of control proposed based on a MPC controller

$$
Q=\operatorname{diag}\left(\mathrm{Q}_{\rho_{1}}, \mathrm{Q}_{\rho_{2}}, \mathrm{Q}_{\theta}, \mathrm{Q}_{\dot{\rho}_{1}}, \mathrm{Q}_{\dot{\rho}_{2}}, \mathrm{Q}_{\dot{\theta}}\right)
$$

\subsubsection{Stiffness modulation}

The stiffness modulation is possible by changing the control parameter Q. During the modulation of the angular stiffness $K_{\theta}$, the mechanism has to conserve a static position $\theta^{d}$. As $K_{\theta}$ depends on the cables tensions (5), an initial and a final desired tensions, respectively $\boldsymbol{\tau}^{i}=\left[\tau_{1}^{i}, \tau_{2}^{i}\right]^{T}$ and $\tau^{f}=\left[\tau_{1}^{f}, \tau_{2}^{f}\right]^{T}$, have to be defined. These tensions are computed by solving (1). The solution has the form [18]:

$$
\tau=\mathbf{W}^{+} \Gamma+\mathbf{N} \lambda
$$

where $\mathbf{W}^{+}$denotes the Moore-Penrose pseudoinverse of $\mathbf{W}, \mathbf{N}=[-L \cos (\theta / 2),-$ $L \sin (\theta / 2)]^{T}$ a basis of $\mathbf{W}$ null space and $\lambda$ is a scalar to be defined.

Since the mechanisms is in an equilibrium position, the generated torque produced by the cables is equal to zero $\Gamma=0$. The bounds of $\lambda$, defined as $\left[\lambda_{\text {min }}, \lambda_{\text {max }}\right]$, can therefore be obtained by solving the inequality:

$$
\boldsymbol{\tau}_{\min } \leq \mathbf{N} \lambda \leq \boldsymbol{\tau}_{\max }
$$

Once the bounds of $\lambda$ are obtained, the tensions $\tau^{i}$ and $\tau^{f}$ could be chosen for given values of $\lambda$ that satisfy (22). Then, using (2), the tensions $\tau^{i}$ and $\tau^{f}$ are converted into initial and final desired cables displacement, respectively $\boldsymbol{\rho}^{i}=\left[\rho_{1}^{i}, \rho_{2}^{i}\right]^{T}$ and $\boldsymbol{\rho}^{f}=\left[\rho_{1}^{f}, \rho_{2}^{f}\right]^{T}$. A desired trajectory, from $\boldsymbol{\rho}^{i}$ to $\boldsymbol{\rho}^{f}$, can therefore be defined in order to modulate $K_{\theta}$.

Since the trajectory of interest is now in terms of the cables displacement, the weight matrix $\mathbf{Q}$ has to be selected with high values for $\mathrm{Q}_{\rho_{1}}, \mathrm{Q}_{\rho_{2}}$ and with small values for the rest of the components. A special attention has to be made with the component $\mathrm{Q}_{\theta}$, because its weight allows to ensure that the orientation of the mechanism will not change during the stiffness modulation. 


\section{Results}

The control approach is evaluated on the developed prototype shown in Fig 1. The mechanism is actuated using 2 DC motors. Polyethylene fibre cables are deployed for motion transmission. 2 force sensors are used to measure the cables tensions. Each sensor is placed between one motor and a spring. Moreover, an optical encoder, with a resolution of $0.225^{\circ}$, is used to measure the angle $\theta$.

Trajectory tracking with low and relatively-high dynamic is first assessed. Three trajectories, going from $90^{\circ}$ to $45^{\circ}$, are created using fifth-order polynomial equation. The duration of these trajectories are respectively $30 s, 5 s$ and $1 s$. The cables tensions limits are set to $\tau_{\min }=4 N$ and $\tau_{\max }=10 \mathrm{~N}$ and the control parameters of $\mathbf{Q}$ are defined in function of the trajectory. For the first trajectory, $Q_{\theta}=8 e 4, \mathrm{Q}_{\dot{\theta}}=50$. For relatively-high dynamic trajectories, $Q_{\theta}=2.1 e 5, \mathrm{Q}_{\dot{\theta}}=8$. The control parameters $Q_{\rho_{1}}=Q_{\rho_{2}}=\mathrm{Q}_{\dot{\rho}_{1}}=\mathrm{Q}_{\dot{\rho}_{2}}=10^{-3}$ and $\mathbf{R}=\operatorname{diag}(9 e 8,9 e 8)$ are set equal for all the trajectories. $H_{p}$ and $H_{u}$ are set equal to 40 with a sampling time of $7 \mathrm{~ms}$. The constrained MPC optimization problem (19) is solved using the Interior point method implemented in the EIgen quadprog solver. The results can be observed in Fig 3.
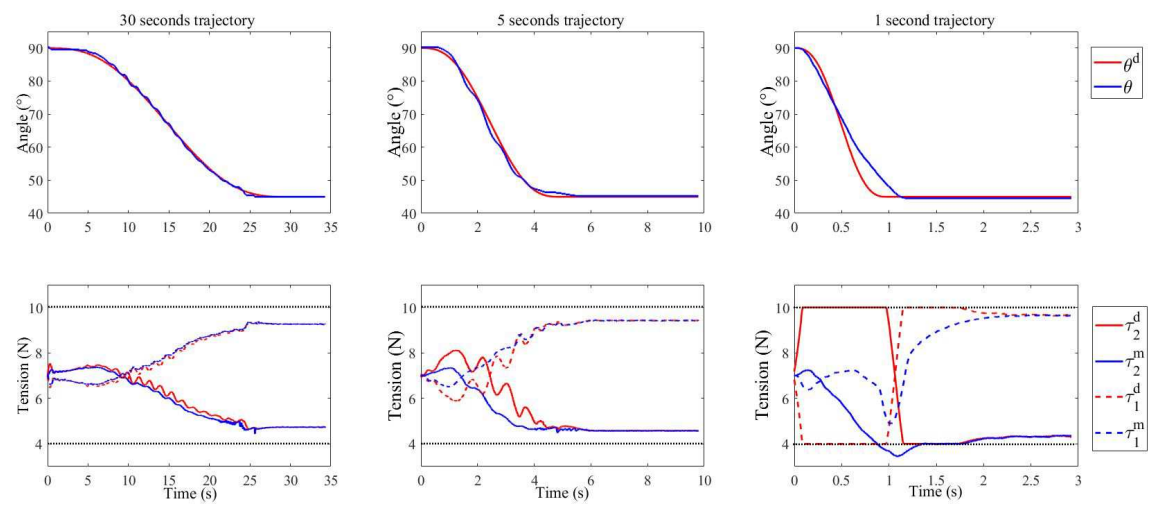

Fig. 3 Trajectory tracking with low and relatively high dynamics.

The root mean square errors (RMSE) of the position tracking for the three trajectories are respectively $0.47^{\circ}, 1.28^{\circ}$ and $4.36^{\circ}$. The static errors is less than or equal to $0.225^{\circ}$ which is the encoder resolution. The obtained results are compared to the

Table 1 Tracking trajectory results for PID controller [10] and the MPC approach.

\begin{tabular}{ccc}
\hline Trajectory & PID & MPC \\
\hline $30 \mathrm{~s}$ & $0.97^{\circ}$ & $0.47^{\circ}$ \\
$5 \mathrm{~s}$ & $3.6^{\circ}$ & $1.28^{\circ}$ \\
\hline
\end{tabular}

literature ones [10], where similar experiments have been conducted using a PID 
controller. Reductions of $51 \%$ and $64 \%$ in the RMSE for the two trajectories, $30 \mathrm{~s}$ and $5 s$, are observed (cf. Table 1). Besides, the RMSE increases when the duration of trajectories decreases. This is mainly due the bandwidth of the tension inner loop, limited actually to $1 s$ of time response. A solution could be to reformulate the MPC problem by changing the control vector $\mathbf{u}$ from motor's torques to motor's angular velocities. Thus, this reformulation eliminates the tension inner loop, and would improve the time response of the system.

A final experiment is performed in order to validate the stiffness modulation of the system. This experiment is divided into two phases. In the first phase, trajectory tracking is performed during $30 \mathrm{~s}$, going from $90^{\circ}$ to $70^{\circ}$. Once the trajectory tracking phase is finished, the stiffness modulation is assessed during $10 s$, while the position of the mechanism is kept at $\theta_{f}=70^{\circ} . \tau^{i}$ is defined as desired tensions at the end of the trajectory tracking. $\tau^{f}$ is defined by setting $\lambda=\lambda_{\max }$. A linear displacement of the cables, going from $\boldsymbol{\rho}^{i}$ to $\boldsymbol{\rho}^{f}$, is therefore defined and sent to the MPC controller. The weights variables of $\mathbf{Q}$ are defined as $\mathrm{Q}_{\rho_{1}}=\mathrm{Q}_{\rho_{2}}=7.2 e 5$, $Q_{\theta}=\mathrm{Q}_{\dot{p}_{1}}=\mathrm{Q}_{\dot{\rho}_{2}}=1, Q_{\dot{\theta}}=10^{-3}$ and $\mathbf{R}=\operatorname{diag}(7 e 7,7 e 7)$. The results are depicted in Fig 4 . During the trajectory tracking, the angular stiffness is quite constant. During

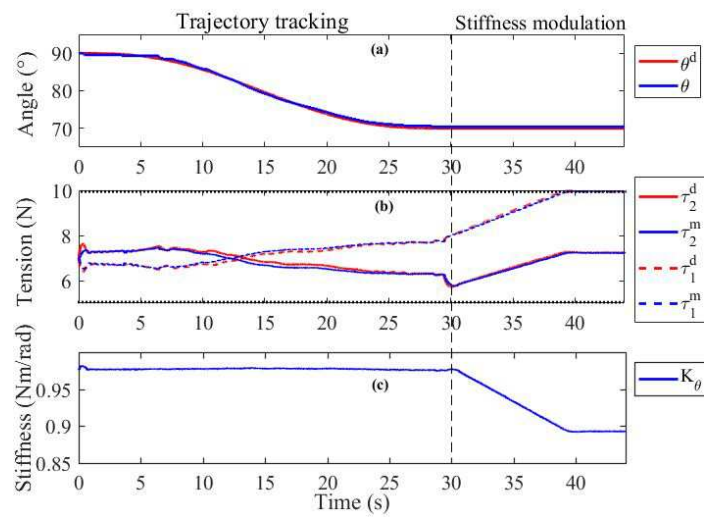

Fig. 4 Results of the trajectory tracking and stiffness modulation experiment

the stiffness modulation, $K_{\theta}$ varies linearly from $0.98 \mathrm{Nm} / \mathrm{rad}$ to $0.89 \mathrm{Nm} / \mathrm{rad}$. This variation is related to the cables tensions variation, as expected in (5) and observed in Fig 4 (b and c). The evolution of the angular stiffness $K_{\theta}$ is inversely proportional to the variation of $\tau$.

\section{Conclusion}

This work presents a linear MPC control strategy for tensegrity mechanisms. The approach allows the position tracking and the stiffness modulation by only chang- 
ing the values of the control parameters $\mathbf{Q}$ and $\mathbf{R}$. A planar cable-driven tensegrity mechanism was used to validate the performance of the proposed controller. The results have shown a static error of less than $0.225^{\circ}$ and good performance in terms of tracking relatively-high and low dynamics trajectories. A reduction of up to $64 \%$ on the RMSE during the trajectory tracking, with respect to literature results, was obtained. Furthermore, the MPC proposed shows a decoupled control of the position and the stiffness.

Future work will be the extension of this approach for spatial tensegrity mechanisms. An interesting perspective would be the proposal of a MPC with the motor's angular velocity as the output control vector, in order to avoid the use of an inner tension control loop.

\section{References}

1. B. Fuller, "Tensile-integrity structures", United States Patent No. 3,063,521, 1962.

2. A. Pugh, "An introduction to tensegrity. Berkeley", University of California Press, Los Angeles, Californie, United States, first edition, 1976.

3. K. Snelson, "Space Frame Structure Made By 3-D Weaving of Rod Members", US Patent 6739937.2004.

4. C. Sultan, "Modeling, design, and control of tensegrity structures with applications", Purdue University, 1999.

5. R. E. Skelton and M. C. De Oliveira, "Tensegrity Systems", Springer, 2009.

6. Ann C. Sychterz, and Ian F. C. Smith, "Deployment and Shape Change of a Tensegrity Structure Using Path-Planning and Feedback Control",Frontiers in Built Environment,2018.

7. C. Paul, F. J. Valero-Cuevas and H. Lip-Son, "Design and control of tensegrity robots for locomotion", IEEE Trans. Robot., pp. 944-957, vol. 22, no 5, 2006.

8. V. Sunspiral, A. Agogino and D. Atkinson, "Super Ball Bot - Structures for Planetary Landing and Exploration", NIAC Phase 2, 2015.

9. M. Furet and P. Wenger, "Kinetostatic Analysis and Actuation Strategy of a Planar Tensegrity 2-X Manipulator", J. Mechanisms Robotics, 2019.

10. Q. Boehler, S. Abdelaziz, M. Vedrines, P. Poignet, P. Renaud, "From modeling to control of a variable stiffness device based on a cable-driven tensegrity mechanism", Mechanism and Machine Theory, Elsevier, pp. 1-12, vol. 107, 2017

11. M. Masic and R. E. Skelton, "Open-loop control of class-2 tensegrity towers", vol. 5383, pp. 298,2004

12. A. Iscen , K. Caluwaerts, et al . "Learning Tensegrity Locomotion Using Open-Loop Control Signals and Coevolutionary Algorithms",Artificial life, vol.21, pp.119-140, 2015.

13. N. Kanchanasaratool and D. Williamson, "Modelling and control of class NSP tensegrity structures", Int. J. Control, vol. 75, no 2, pp. 123-139, 2002.

14. D. Hustig-Schultz and V. SunSpiral and M. Teodorescu. "Morphological design for controlled tensegrity quadruped locomotion", IEEE/RSJ International Conference on Intelligent Robots and Systems (IROS) ,pp. 4714-4719.2016.

15. A.S. Wroldsen and M.C. de Oliveira and R.E. Skelton,"Modelling and control of non-minimal non-linear realisations of tensegrity systems", 2009.

16. Andrew P. Sabelhaus et al.”Model-Predictive Control with Reference Input Tracking for Tensegrity Spine Robots" in arXiv:1806.08868, 2019.

17. J. Maciejowski, Predictive Control with Constraints. Prentice Hall. 2002

18. M. Gouttefarde, J. Lamaury, C. Reichert, T. Bruckmann, ”A Versatile Tension Distribution Algorithm for n-DOF Parallel Robots Driven by n+2 Cables," IEEE Transactions on Robotics, Vol. 31, No. 6, pp. 1444-1457, 2015. 